\title{
Intestinal Dysbiosis and Premature Delivery: Relationship Between Gestation, Type of Delivery and Maturation of the Neonatal Intestinal Flora
}

\section{Disbiosis Intestinal y Parto Prematuro: Relación Entre Duración de la Gestación, Tipo de Parto, Maduración de la Flora Intestinal}

XVIII International Seminar on Health, Food and Human Nutrition

Corresponding Author:

Iván Enrique Naranjo Logroño

naranjometropolitana@hotmail.com

Published: 9 September 2021

Production and Hosting by

Knowledge E

(a) Iván Enrique Naranjo Logroño et al. This article is distributed under the terms of the Creative Commons Attribution License, which permits unrestricted use and redistribution provided that the original author and source are credited.

\section{Neonatal}

Iván Enrique Naranjo Logroño ${ }^{1}$, Anthony Alfonso Naranjo Coronel ${ }^{2}$, Ashley Carolina Cuzco Macías ${ }^{1}$, and Cristian Alberto Zumárraga Pozo ${ }^{1}$

${ }^{1}$ Carrera de Medicina, Facultad de Salud Pública, ESPOCH, Riobamba, Chimborazo, Ecuador
${ }^{2}$ Médico Cirujano, COLPOMED Centro, Hospital del Día, Riobamba, Chimborazo, Ecuador

\section{Abstract}

Introduction: The imbalance of the microorganisms found in the intestine, is called intestinal dysbiosis, this condition allows the spread or increase of pathogenic bacteria. Objective: To synthesize the paper that maternal intestinal dysbiosis plays in the pathogenesis of preterm birth and the correlation between pregnancy duration, type of delivery and maturation of neonatal intestinal flora. Methods: A bibliographic search was carried out in the following search engines: SCIENCE DIRECT, ELSEVIER, COCHRANE, SCOPUS, MEDLINE, LILACS, CINAHL, PUBMED, SCIELO, EBSCO. Results: 43 articles were found, of which 35 articles were used that contributed transcendental information to this review, and 8 articles that did not meet the criteria were excluded. Discussion: The variation of the balance between the bacteria in the intestine can cause, what is known as maternal inflammatory bowel disease, which is related to premature birth and other conditions Conclusion: Maternal intestinal dysbiosis can cause inflammatory reactions in the uterus that lead to premature delivery, however, more studies are needed to clarify their relationship.

Keywords: dysbiosis, premature delivery, pregnancy, caesarean section, newborn.

\section{Resumen}

Introducción: La pérdida del equilibrio de los microorganismos que se encuentran en el intestino, es llamado disbiosis intestinal, esta condición permite la propagación o el aumento de bacterias patógenas. Objetivo: Sintetizar el papel que desempeña la disbiosis intestinal materna en la patogénesis del parto prematuro y la correlación que existe entre la duración de la gestación, el tipo de parto y la maduración de la flora intestinal neonatal. Métodos: Se realizó una búsqueda bibliográfica en los siguientes buscadores: SCIENCE DIRECT, ELSEVIER, COCHRANE, SCOPUS, MEDLINE, LILACS, CINAHL, PUBMED, SCIELO, EBSCO. Resultados: Se encontraron 43 artículos de los cuales se utilizaron 35 artículos que aportaron información transcendental a esta revisión, y se excluyeron 8 artículos que no cumplieron con los criterios planteados. Discusión: La variación del equilibrio entre las bacterias en el intestino puede 
originar, lo que se conoce como enfermedad inflamatoria materna del intestino, misma que se relaciona con el parto prematuro y a otras afecciones. Conclusiones: La disbiosis intestinal materna puede causar reacciones inflamatorias en el útero que conducen a parto prematuro, sin embargo, son necesarios más estudios para aclarar su relación.

Palabras Clave: disbiosis, parto prematuro, gestación, cesárea, neonato.

\section{Introducción}

La unidad materno fetal saludable está colonizada con microorganismos y este es un requisito previo para la maduración inmune, así como para la homeostasis metabólica y hormonal. La vida intrauterina, conjuntamente con el primer año de vida, constituyen etapas esenciales para el desarrollo y funcionamiento óptimo del sistema inmunológico. Por estas razones, los eventos negativos de la vida temprana representan un riesgo especial para el desarrollo somático y psicomotor [1]. La pérdida del equilibrio de los microorganismos que se encuentran en el intestino, es llamado disbiosis intestinal, esta condición permite la propagación o el aumento de bacterias patógenas.

Pueyo y Mach [2] la evidencia respalda la hipótesis de que la disbiosis materna podría actuar como desencadenante del parto prematuro [3-5]. Además la colonización aberrante se asocia con efectos nocivos y puede tener consecuencias a largo plazo que conducen a distintas patologías, como obesidad, enfermedad inflamatoria intestinal (EII), diabetes, síndrome metabólico, entre otras [5]. Sin embargo, la colonización inicial de la microbiota humana y el impacto de la salud materna en la microbiota neonatal al nacer todavía no se conoce con exactitud [6].

Esta revisión tiene como objetivo sintetizar el papel de la disbiosis intestinal de la madre en la patogénesis del parto prematuro y la relación que existe entre la duración de la gestación, el tipo de parto y la maduración de la flora intestinal neonatal.

\section{Metodología}

Se realizó una búsqueda bibliográfica referente a la disbiosis intestinal y su repercusión en el parto prematuro, a más de ello, se realizó un análisis acerca de la relación entre la duración de la gestación, el tipo de parto y la maduración de la flora intestinal del neonato, en los siguientes buscadores: SCIENCE DIRECT, ELSEVIER, COCHRANE, SCOPUS, MEDLINE, LILACS, CINAHL, PUBMED, SCIELO, EBSCO. Para ello, se empleó el método analítico sintético; las palabras claves empleadas fueron: Disbiosis, parto prematuro, gestación, cesárea, neonato, entre otros.

La investigación bibliográfica se realizó en el periodo comprendido entre octubre 2019 a febrero 2020. Se incluyeron artículos originales, revisiones sistemáticas, metaanálisis, ensayos clínicos controlados y randomizados con información relevante acerca del tema, en los idiomas de español e inglés, desde el año 2013 hasta el 2019. Se excluyeron aquellos artículos que no aportaban información acorde al tema. 


\section{Resultados}

Se encontraron 43 artículos científicos acerca de la disbiosis intestinal y su repercusión en el parto prematuro; y la relación entre la duración de la gestación, el tipo de parto y la maduración de la flora intestinal del neonato; de los cuales se utilizaron 35 artículos que aportaron información transcendental a esta revisión, y se excluyeron 8 artículos que no cumplían con los criterios planteados.

\section{Discusión}

El parto prematuro comprende una gran cantidad de causas diferentes, entre estos, la inflamación y la infección son los principales desencadenantes del parto prematuro y representan la mitad de todos los casos, al menos [1]. Existen muchos factores impulsores de la disbiosis intestinal, sin embargo, se ha asociado de manera más frecuente al estrés, a la higiene, intolerancia a ciertos azucares y el uso arbitrario de antibióticos. En el embarazo la flora intestinal puede verse afectada por los cambios hormonales, es así que las causas en este estado aumentan de forma importante, siendo necesario tomar mayores precauciones [7]. Otro de los factores que pueden desencadenar la disbiosis intestinal en la madre es la incorrecta alimentación durante el estado gestante, pues una ingesta rica o abundante en grasa y con un contenido de fibra muy bajo, puede asociarse con esta alteración del equilibro de la microbiota intestinal, predisponiendo a la madre a una condición de obesidad [8]. En una edad avanzada también existen cambios en la microbiota, tanto en su variedad de bacterias presentes y en la disminución de ciertos microrganismos [9].

\subsection{Disbiosis intestinal y el embarazo}

La microbiota en todos los seres humano es importante y aún más cuando se trata de una mujer en estado de gestación; al existir varias etapas con cambios generales y hormonales durante este periodo en todo el cuerpo de la madre, se puede generar alteraciones en éstas, siendo necesario el control minucioso de su salud [10]. La microbiota materna contribuye al parto prematuro, en lugar de la infección de un microbio específico [11].

Es necesario conocer de manera general los cambios en torno a la microbiota existente en varias partes de la mujer, que colonizarán al bebé durante y después del parto. En primer lugar, la microbiota vaginal, pues durante el embarazo, pasa por diferentes etapas relacionadas con la función endocrina, para preparar a la vagina con lactobacilos que serán trasferidos al bebé durante el parto. Por otro lado, la leche materna también cuenta con la presencia de microorganismos como Grampositivos y Gramnegativos necesarios para mantener sano al sistema digestivo del neonato, aportando de manera continua bacterias durante la lactancia, es así que puede aportar a la flora intestinal una cantidad aproximada entre 100 mil y 10 millones de bacterias por día. Aunque la microbiota vaginal es importante para el desarrollo del neonato durante y después del parto, es necesario considerar que existen alteraciones de 
igual tipo en la microbiota intestinal, que, si se produce durante el embarazo y no se controla adecuadamente, con la alimentación, suplementos y cuidados específicos, puede provocar complicaciones severas [12, 13].

La microbiota intestinal al igual que los demás, varía conjuntamente con el avance del embarazo, pues existe un aumento de bacterias patógenas como proteobacteria y actinobacteria, y a la par disminuyen las bacterias benéficas como las bacteroidetes y firmicutes, condición presentada en los últimos tres meses de gestación [14]. Cuando hay alguna alteración importante de la microbiota intestinal en una mujer en estado de gestación, sin el adecuado control o tratamiento, ya sea presentado en cualquier etapa, los efectos pueden aumentar el riesgo de un parto prematuro, preeclampsia, restricción del crecimiento intrauterino (RCIU) y abortos espontáneos [15]. En relación a este último, el intercambio de nutrientes mediante el cordón umbilical, modifica la microbiota intestinal, los microorganismos invadan el mismo y puede generar inflamación del entorno y producirse un aborto espontaneo [16].

La variación del equilibrio entre las bacterias en el intestino puede originar, lo que se conoce como enfermedad inflamatoria materna del intestino, misma que se asocia con el parto prematuro y otras afecciones. Básicamente la reducción de la diversidad de la microbiota intestinal de la mujer gestante, entre ellas la disminución de Bifidobacterium, Streptococcus y Clostridium, son las causantes de esta disbiosis intestinal [17].

\subsection{Tiempo de gestación}

Para comenzar con el desarrollo de este factor es necesario comprender que el tiempo de gestación, es tan importante como los cuidados administrados a la madre durante el embarazo, ya que, aunque no esté totalmente claro, existen investigaciones en curso que mencionan que la obtención de una microbiota prematura es una causante influyente de diversas afecciones [18]. Los recién nacidos prematuros, se ven cada vez más afectados por las complicaciones asociadas con la disminución de la edad gestacional. Las complicaciones de la prematuridad incluyen la maduración deteriorada de la microbiota intestinal, el tracto digestivo y el sistema inmunitario [19].

Si se compara los niveles de bacterias patógenas y benéficas entre un neonato nacido de forma prematura y uno a término, en el primero la diversidad de la flora intestinal es muy baja y el aumento de las bacterias de naturaleza patógena es evidente, y por ende la presencia de Bifidobacterium y Bacteriodes es muy baja colonizándose en los próximos 10 días aproximadamente [20]. Durante las primeras semanas postparto, la microbiota intestinal del recién nacido prematuro con edad gestacional de 23 a 30 semanas varía con predominio de Escherichia, Klebsiella, Enterococcus y Staphylococcus [11].

\subsection{Tipo de parto}

Este medio de colonización se cree, es el más determinante, pues durante el parto, el neonato se pone en contacto con las bacterias más predominantes del medio, ya que estudios determinan que el tipo de microorganismos que se encuentran en la flora del 
intestino inicial del bebé dependen en gran medida del canal o método de concepción [6].

Los neonatos nacidos bajo el mecanismo de parto tipo vaginal son colonizados gracias a las bacterias de las heces y vagina de la madre, siendo conformada por fimicutes, lactobacilos y bifidobacterias de manera abundante $[19,20]$. Se descubrió que las composiciones microbianas vaginales difieren según la edad gestacional, y las comunidades en las últimas etapas del embarazo se parecen a las del estado no embarazado [6].

\subsection{Desarrollo de la flora intestinal del neonato}

Los recién nacidos a término normales primero son colonizados por Streptococcus, Staphyococcus, Escherichia coli, Lactobacillus y Enterobacter. A medida que estas especies consumen oxígeno, permiten la posterior colonización de especies bacterianas anaeróbicas, principalmente Clostridia, Bifidobacterium y miembros de Firmicutes phyla, sin embargo la maduración de la flora intestinal finaliza entre los 2 y 4 años de edad [21]. La microbiota intestinal en los bebés prematuros se desarrolla de manera diferente. Los bebés prematuros se alimentan enteralmente antes de lo previsto por la naturaleza y con frecuencia reciben alimentos fortificados. Tienen un sistema inmunitario inmaduro, además, tienen una mayor cantidad de bacterias potencialmente patógenas como E. coli, Staphylococcus y Klebsiella [22]. También se ha comprobado, que mientras más anormal sea el microbioma en los lactantes de la Unidad de Cuidados Intensivos Neontatal, es más probable que experimenten un retraso en el crecimiento incluso a los 4 años de edad [23].

La salud o equilibrio de la flora intestinal del neonato dependen de varios factores que están presentes desde el embarazo y nacimiento, pues la duración de la gestación, la vía de parto, el tiempo del consumo de leche materna o la elección de la ingesta de formula infantil y la alimentación adicional, son patrones que han demostrado la influencia de las características de la microbiota intestinal, incluso la variación de ésta puede darse por el estrés o la ubicación demográfica [8, 24].

Para la completa formulación de la microbiota se ha establecido que se sigue varias fases continuas (Tabla 1) [17]. La falta de maduración de una flora intestinal de un neonato prematuro es muy similar a la flora intestinal de un neonato desnutrido, siendo éste un desencadenante para el crecimiento deficiente del niño. Sin embargo, una adecuada alimentación y cuidado a temprana edad puede ayudar a la corrección de esta deficiencia estimulando el crecimiento y desarrollo de forma saludable $[25,26]$.

\subsection{Funciones de la flora intestinal}

La importancia de un desarrollo adecuado de la microbiota intestinal radica en su influencia sobre diferentes sistemas, además de funcionar como barrera a la mucosa intestinal, ya que favorece en la generación de mucosidad que recubre la pared interna del intestino, pues ayuda a la retención de ciertos microorganismos dañinos para el organismo que luego son expulsados gracias al suave movimiento intestinal, de 


\section{Table 1}

Fases del desarrollo de la microbiota intestinal.

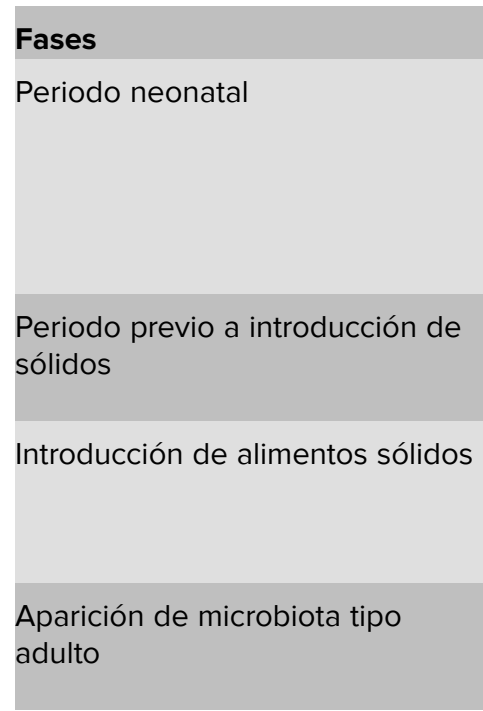

Instalación
Dos primeras semanas de vida
Final de la segunda semana de
vida hasta el inicio de la
ablactación
Etapa de introducción de dieta
con suplementos sólidos
Etapa completa de la ablactación
(alrededor de 2-3 años)

Características
De inicio, E. coli/Streptococcus
influida por tipo de alimentación
-Lactancia materna:
Bifidobacterias
-Lactancia artificial:
Enterobacterias
Si lactancia materna, colonización
con bifidobacterias hasta un $90 \%$,
con producción de heces ácidas
Microbiota similar a niños
alimentados con lactancia
artificial Aparecen E. coli,
Streptococcus y Clostridium
Se constituye complejo
ecosistema intestinal con
microbiota de

Tabla que expresa la variedad de microbiota que se puede adquirir a lo largo de diferentes etapas del desarrollo.

Tomado de: Guillot CC. Microbiota intestinal y salud infantil, 2018.

igual forma genera enzimas que actúan como bactericidas mismas que controlan la proliferación de bacterias ajenas al sistema [8].

La microbiota intestinal también ayuda a varias funciones referentes al metabolismo, pues intervienen en la generación de nutrientes desde compuestos que no se pueden digerir provenientes de la alimentación, además cuenta con la función de absorber agua y minerales. Si se hace énfasis en la alimentación, ayuda en la digestión de carbohidratos en forma de lactosa y sintetiza a la par ciertas vitaminas del grupo B, como la tiamina (B1), la piridoxina (B6), la cobalamina (B12), además del ácido nicotínico y el fólico [8].

\subsection{Factores que influyen en el desarrollo de la flora intestinal}

La flora bacteriana o microbiota intestinal se considera como un órgano más que se forma o adquiere al momento del parto, sin embargo, la evidencia actual demuestra que la microbiota de varios sitios del organismo de la madre como la placenta, la boca o el intestino delgado intervienen en el desarrollo de la flora intestinal del feto. Aun así, a partir del alumbramiento se tiene claro que existen factores que influyen en la riqueza o calidad del ambiente microbiano [27]. Por esta razón es importante mencionar y explicar de forma general los factores que determinan el buen desarrollo y las variantes que se pueden presentar en base a ellos manifestando una buena salud o por el contrario enfermedad.

A pesar de contar con una flora intestinal diferente y única cada individuo, es fundamental el mantener un equilibrio entre los microorganismos que se encuentran en el intestino, ya que su diversidad aporta funciones que pueden favorecer o atacar 
al mismo organismo. El control y cuidado desde la etapa de gestación puede influir de manera importante en todo lo que refiere el flujo adecuado de electrolitos, agua y nutrientes a través de las paredes del intestino y mediante un proceso osmótico del epitelio intestinal. Si éstas fallan, aparece la translocación bacteriana, misma que altera su funcionamiento general [28]. En la primera etapa de colonización del neonato, luego del nacimiento, la microbiota intestinal es muy vigorosa y cambiante, pues a partir del parto, el primer contacto con la microbiota de la piel de la madre, la manera de alimentación y otros cuidados como la administración de antibióticos es determinante en el desarrollo de la misma [28].

El desarrollo, función y características de la flora intestinal son definidas gracias al canal de parto, la microbiota de la madre, la ingesta temprana de antibióticos y la forma de alimentación. Además, la alimentación y duración de la etapa de lactancia son indicadores del correcto desarrollo de las bacterias del intestino en el neonato $[17,25]$.

\subsection{Forma de alimentación}

El desarrollo de la flora intestinal del neonato está determinado de forma importante gracias a la forma de alimentación, siendo factible en mayor grado la alimentación por medio de la lactancia materna, pues se ha encontrado una cantidad importante de Bifidobacterias y lactobaccillus en la flora intestinal, mientras que en los neonatos alimentados mediante formula infantil, desarrollan en gran medida bacterias del tipo Bacteroides, Enterobacterias y Streptococus [29].

La transferencia de la flora bacteriana desde el seno al bebé, se da mediante la administración continua por vía oral, siendo incluso funcional con características nutricionales y con ciertas propiedades de prebióticos, sin embargo, la composición de este tipo de microbiota depende en gran medida de la etapa de lactancia y su duración, ya que cambia la variedad de microorganismos en el calostro o en la etapa inicial de lactancia además depende de la alimentación y salud general de la madre [30].

La vía enteromamaria, es la encargada de llevar bacterias seleccionadas desde el tracto intestinal materno hasta la leche que va a ingerir el nuevo ser (Gráfico 1), evidenciándose la trascendencia de la adecuada alimentación de la madre durante el embarazo y también en la lactancia. De igual forma durante esta etapa se ha observado una importante translocación bacteriana desde el intestino hasta los ganglios linfáticos mesentéricos para luego dirigirse a la glándula mamaria, generando de esta forma el complemento y enriquecimiento de la leche materna. Se incluye el traspaso de células del sistema inmunológico como inmunoglobulinas, citosinas, probióticos y prebióticos y mucho ADN bacteriano libre, beneficiosos para el neonato, gracias a la lactancia materna [31, 32].

Varias investigaciones han definido que la administración de suplementos en base a probióticos y prebióticos, ayudan en el desarrollo y la colonización beneficiosa de la flora del intestino del neonato, pues hasta 6 meses después de la alimentación de estos suplementos se ha mantenido la presencia en el intestino de los mismo [33]. Esta clase de suplementación es ideal para niños alimentados con formula infantil, llegando 
a alcanzar resultados favorables en comparación con bebes alimentados mediante la lactancia materna [34].

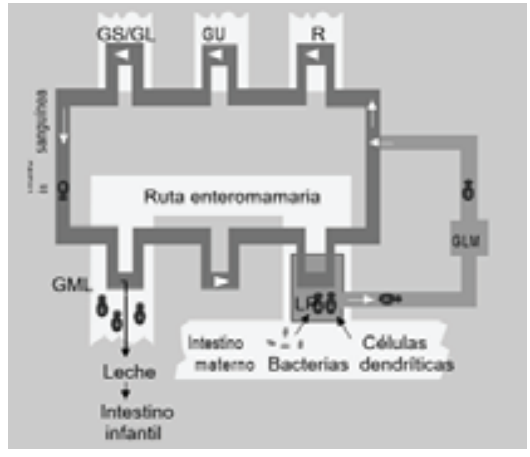

Figure 1

Ingreso de bacterias intestinales a la leche materna por la vía enteromamaria. GLM: Ganglio linfático mesentérico; GML: Mucosa de la glándula mamaria lactantes; GSL: Mucosa de las glándulas salivales y lacrimales; GU: Mucosa del tracto genitourinario; LP: Lámina propia; $R$ : Mucosa del tracto respiratorio. Tomado de: Navarro-Cáceres W, La lactancia materna y sus propiedades microbioinmunológicas, 2011.

De manera general la flora intestinal, tanto en la madre como en el neonato, son fundamentales en el correcto funcionamiento del organismo, desde la etapa de gestación para evitar complicaciones propias del desequilibro de la microbiota intestinal materna y en el desarrollo y maduración de la flora intestinal del bebé. Al no contar con los cuidados debidos, puede generarse una disbiosis intestinal y por ende causar partos prematuros, por ello, se requiere una alimentación completa y saludable durante la etapa gestacional. Además, las evidencias de interacciones entre bacterias intestinales con el feto durante el embarazo, puede llevar a la translocación bacteriana produciendo disbiosis y daños al útero, siendo un factor de riesgo para el parto prematuro, enfatizando al control y cuidado antes, durante y después de la etapa gestacional [35].

\section{Conclusiones}

La microbiota saludable es un mediador crítico para mantener la salud del feto, por lo que la disbiosis intestinal materna puede causar reacciones inflamatorias en el útero que conducen a parto prematuro. Sin embargo, son necesarios más estudios para aclarar su relación.

Es imprescindible destacar que una perspectiva centrada en la microbiota tiene el potencial de innovar la forma en que se desarrollan y administran las intervenciones para la prevención del parto prematuro.

\section{Agradecimiento}

Nuestro agradecimiento a la Escuela Superior Politécnica de Chimborazo, a la Facultad de Salud Pública y al XVIII Seminario Internacional de Salud, Alimentación, y Nutrición 
Humana, a su comité organizador por su esfuerzo colaborativo en la difusión de investigación científica.

\section{Conflictos de Interés}

Los autores declaramos no tener ningún conflicto de interés.

\section{Limitaciones de Responsabilidad}

Los autores declaramos que toda la información recopilada en el presente documento es de nuestra responsabilidad y no de la institución en la que laboramos.

\section{Fuentes de Financiación}

La financiación del presente documento proviene de los mismos autores.

\section{References}

[1] Staude B, Oehmke F, Lauer T, et al. The Microbiome and preterm birth: A change in paradigm with profound implications for pathophysiologic concepts and novel therapeutic strategies. Biomed Res Int. 2018.

[2] Pueyo B, Mach N. Disbiosis intestinal en enfermos de Crohn pediátricos blanca pueyo. Nutr Hosp. 2013;28(6):1820-8.

[3] Wang J, Zheng J, Shi W, et al. Dysbiosis of maternal and neonatal microbiota associated with gestational diabetes mellitus. Gut. 2018;67(9):1614-25.

[4] Baldassarre ME, Di Mauro A, Capozza M, et al. Dysbiosis and prematurity: Is there a role for probiotics? Nutrients. 2019;11(6).

[5] Shiozaki A, Yoneda S, Yoneda N, et al. Intestinal microbiota is different in women with preterm birth: Results from terminal restriction fragment length polymorphism analysis. PLoS One. 2014;9(11):e111374.

[6] Nuriel-Ohayon M, Neuman H, Koren O. Microbial changes during pregnancy, birth, and infancy. Front Microbiol. 2016;7( JUL).

[7] Guarner F. Cuando la microbiota intestinal pierde su equilibrio. Miami; 2016.

[8] Rodríguez J, Sobrino O, Marcos A, et al. ¿Existe una relación entre la microbiota intestinal, el consumo de probióticos y la modulación del peso corporal? Nutr Hosp. 2013;28:587-601.

[9] Catassi C. El microbioma intestinal: un universo en desarrollo. J Heal care prof. 2015;1.

[10] Del Car M; Giglio B. Microbiota y ecosistema intestinal. Probióticos ¿por qué y para qué? Rev Hosp Niños. 2014;56(255).

[11] Valentine G, Chu DM, Stewart CJ, Aagaard KM. Relationships between perinatal interventions, mater nal-infant microbiomes, and neonatal outcomes. Clin Perinatol. 2018;45.

[12] Álvarez-Calatayud G, Suárez E, Rodríguez JM, Pérez-Moreno J. La microbiota en la mujer; aplicaciones clínicas de los probióticos. Nutr Hosp. 2015;32:56-61.

[13] Chan YK, Estaki M, Gibson DL. Clinical consequences of diet-induced dysbiosis. Ann Nutr Metab. 2013;63(2):28-40.

[14] Pammi M, Cope J, Tarr PI, et al. Intestinal dysbiosis in preterm infants preceding necrotizing enterocolitis: A systematic review and meta-analysis. Microbiome. 2017;5(1):31.

[15] Gonzalez G. Terapia Médico-Nutricia durante el embarazo y enfermedad renal crónica. 2017.

[16] Herrera MDA. Preterm delivery and intimacy during pregnancy: Interaction between oral, vaginal and intestinal microbiomes. Medwave. 2015 May;15(4):e6144.

[17] Robertson RC, Manges AR, Finlay BB, Prendergast AJ. The Human microbiome and child growth First 1000 Days and Beyond. Trends Microbio. 2019;27(2):131-47. 
[18] Warner BB, Deych E, Zhou Y, et al. Gut bacteria dysbiosis and necrotising enterocolitis in very low birthweight infants: A prospective case-control study. Lancet. 2016;387(10031):1928-36.

[19] Henderickx JGE, Zwittink RD, Van Lingen RA, Knol J, Belzer C. The preterm gut microbiota: An inconspicuous challenge in nutritional neonatal care. Frontiers in Cellular and Infection Microbiology. 2019;9.

[20] Guillot CC. Microbiota intestinal y salud infantil Intestinal microbiota and child health. Rev Cubana Pediatr. 2018;90.

[21] Denning NL, Prince JM. Neonatal intestinal dysbiosis in necrotizing enterocolitis. Mol Med. BioMed Central Ltd.; 2018;24:1-10.

[22] Elgin TG, Kern SL, McElroy SJ. Development of the neonatal intestinal microbiome and its association with necrotizing enterocolitis. Clinical Therapeutics. 2016;38:706-15.

[23] Universidad del Sur de Florida. Gut microbiome of premature babies is associated with stunted growth. ScienceDaily. 2019.

[24] Masís BA, Ivancovich EG. Generalidades de la microbiota intestinal y su rol en algunas patologías. Rev Clin Esc Med. 2013;3(12).

[25] Ihekweazu FD, Versalovic J. Development of the pediatric gut microbiome: Impact on health and disease. Am J Med Sci. 2018;356(5):413-23.

[26] Serrano HCA, Leon RMA, Harris Diez PR. Desarrollo de la microbiota gastrointestinal en lactantes y su rol en salud y enfermedad. ARS med. 2016;41(1):35-43.

[27] Daza W, Dadán S, Higuera M. Microbiota intestinal y sobrecrecimiento bacteriano. CCAP; 2015.

[28] Mena V. El intestino en el proceso salud/enfermedad. Rev Cuba Pediatr. 2017;89(2):105-7.

[29] Zamudio-Vázquez VP, Ramírez-Mayans JA, Toro-Monjaraz EM, et al. Importancia de la microbiota gastrointestinal en pediatría. Acta Pediatr Mex. 2017;38(1):49-62.

[30] Brunser T. O. El desarrollo de la microbiota intestinal humana, el concepto de probiótico y su relación con la salud humana. Rev Chil Nutr. 2013;40(3):283-9.

[31] Blázquez R, Cuchi E, Martin C, Ruiz P. Diagnóstico microbiológico de la infección por el virus del papiloma humano. $1^{\text {st }}$ ed. Cercenado E, Canton R, editors. Enferm Infecc Microbiol Clin.; 2017.

[32] Hernández D, José E, Cabeza G, Niurka D, Castañeda S. La microbiota intestinal en el desarrollo del sistema inmune del recién nacido. Rev Cuba Pediatr. 2014;86(4):502-13.

[33] García E, Angulo E, Carrillo S, et al. Eficacia y seguridad de los probióticos en el recién nacido pretérmino. Rev Med MD. 2014;5:238-47.

[34] Lopez M. Influencia de la gestación, el parto y el tipo de lactancia sobre la microbiota intestinal de neonato. Acta Pediatr Esp. 2014;72(2):37-42.

[35] Cantón R, Del Campo R, Mira A, Monsó E, Carracedo Á, Lapunzina P. Informes anticipado Microbiota. 2018. 